\title{
PRATIQUES CULTURELLES ET CIRCULATION EN EUROPE
}

\author{
LES « LUSODESCENDANTS ${ }^{1}$ »
}

Irène Dos SANTOS

RÉSUMÉ : Le regard ethnologique, porté par l'auteur sur les pratiques de circulation entre la France et le Portugal de jeunes d'ascendance portugaise et de jeunes Portugais de France, révèle l'agencement de ressources matérielles et symboliques plurielles. Au-delà de la dimension économique et politique dans lesquelles s'insèrent ces pratiques, le processus identitaire dans lequel elles s'intègrent pose des questions, notamment dans le contexte de l'Union européenne. L'auteur montre ici comment, en puisant des éléments de l'histoire migratoire familiale, de l'histoire nationale et de la culture portugaise, des descendants de migrants économiques européens tentent de dépasser le dilemme identitaire que représente la « double culture », ainsi que les contraintes sociales et culturelles imposées par leurs aînés.

MoTS-CLÉS : immigration, France/Portugal, « lusodescendants », identité, mémoire collective, retour.

ABSTRACT: In the present study the author examines the migratory practices of movement between France and Portugal of young French of Portuguese ancestry, as well as of young Portuguese living in France. The study of these practices from an ethnological point of view sheds light on the interaction of material resources and the multifaceted symbolic resources associated with them. Besides the economic and political dimension in which these practices are grounded, the identity-forming process of which they are a part raises a number of questions - especially in the context of the European Union. The author shows how, by using elements drawn from the family migratory history, natural history and Portuguese culture, the descendents of Europeans who migrated for economic reasons are now trying to go beyond the dilemma of an identity posed by their "double culture», as well as the social and cultural constraints imposed on them by their elders.

KEYWORDS : immigration, France/Portugal, « lusodescendants », identity, collective memory, return.

1. Dénomination des enfants de migrants portugais expatriés, dont fait usage l'État portugais (sous la forme Luso-descendente) ainsi qu'une partie du réseau associatif portugais dans le monde. Nous utiliserons la forme « lusodescendant » choisie en France par nos informateurs. 
ZuSAMMENFASSUNG : In der vorliegenden Studie betrachtet der Autor die migratorischen Praktiken junger Franzosen portugiesischer Abstammung, sowie junger in Frankreich lebender Portugiesen, die zwischen Frankreich und Portugal pendeln. Von einem ethnologischen Standpunkt aus wirft die Studie dieser Praktiken Licht auf die Interaktion materieller Mittel und der mit ihnen zusammenhängenden symbolisch vielschichtigen Ressourcen. Über die wirtschaftliche und politische Dimension, auf die sich diese Praktiken begründen, hinaus, wirft der identitätsformende Prozess eine Reihe von Fragen auf, vor allem im Zusammenhang mit der Europäischen Gemeinschaft. Indem der Autor Elemente der familiären Migrationsgeschichte, der nationalen Geschichte sowie der portugiesischen Kultur benutzt, zeigt er hier, wie Nachkommen europäischer Emigranten, die aus ökonomischen Gründen ausgewandert sind, versuchen, das identitäre Dilemma der «Doppel-Kultur» sowie die sozialen und kulturellen Einschränkungen, die ihnen von ihren unmittelbaren Vorfahren aufgezwungen werden, zu überwinden.

STICHWÖRTER : Einwanderung, Frankreich/Portugal, « lusodescendants », Identität, Kollectivgedächtnis, Rückkehr.

Irène Dos SANTOS, née en 1975, est doctorante à l'École des hautes études en sciences sociales (Paris), rattachée au laboratoire d'Anthropologie sociale du Collège de France. Elle est boursière de la Fundação para a Ciência e a Tecnologia. Elle mène sa recherche en France et au Portugal auprès de jeunes d'ascendance portugaise issus de l'émigration économique des années 1960 et 1970. Elle s'intéresse à la diversité des liens, collectifs et individuels, maintenus avec le Portugal, fondés sur des représentations collectives du pays et de sa culture, à la transmission et à la reconstruction d'une mémoire collective dans ce contexte migratoire européen.

Adresse : Laboratoire d'anthropologie sociale, 52 rue du Cardinal-Lemoine, F-75006 Paris.

Courrier électronique : irenedossantos@msn.com 
«Le Portugal se vit "au-dedans", dans une sorte d'isolement sublimé, tout en se montrant "au-dehors" comme l'exemple même des peuples à vocation universelle, allant jusqu'à disperser son corps et son âme dans le monde entier [...] Un tel peuple, à l'aise partout dans le monde comme s'il était chez lui, en fait ne connaît pas vraiment de frontières car il n'a pas d'extérieur. »

Eduardo LOURENÇO ${ }^{2}$

À l'aube du $\mathrm{XXI}^{\mathrm{e}}$ siècle, le nombre de Portugais expatriés est estimé à plus de 4 millions d'individus, pour une population de 10 millions de Portugais vivant dans leur pays. La migration est une donnée structurelle dans l'histoire de ce pays. Les migrations portugaises se sont développées à la suite des découvertes maritimes, à partir du $\mathrm{XV}^{\mathrm{e}}$ siècle, jusqu'à nos jours : d'une nation colonisatrice, le Portugal est devenu une nation émigrante ${ }^{3}$.

Depuis 1980, les émigrés portugais et leurs descendants composent les «Communautés portugaises », prolongement de la nation hors frontières et symbole du caractère cosmopolite de la culture portugaise ${ }^{4}$. En Europe, leur proximité géographique avec le Portugal a engendré une pratique de circulation annuelle entre pays de résidence et pays d'origine : une mobilité géographique allant de pair avec une circulation de biens matériels, culturels et identitaires ${ }^{5}$. L'étude ethnographique des liens perpétués et construits par des descendants de migrants économiques européens nous conduit à nous interroger non plus

2. LOURENÇO, 1997, p. 8 et 11.

3. Peuplement des archipels atlantiques à partir du début $\mathrm{du} \mathrm{XV}^{\mathrm{e}}$ siècle, migrations vers l'Orient et l'océan Indien au XVI $I^{\mathrm{e}}$ siècle, départs de colons vers le Brésil qui s'intensifient au milieu du $\mathrm{XVI}^{\mathrm{e}}$ siècle, et émigration forcée de Juifs vers les dernières décennies du $\mathrm{XVI}^{\mathrm{e}}$ siècle et au cours des premières du XVII ${ }^{\mathrm{e}}$ siècle. Dès la fin du XVII ${ }^{\mathrm{e}}$ siècle, des espaces colonisés (Madère, les Açores) deviennent des foyers d'intense émigration vers le Brésil, le Mozambique, et plus tard vers l'Amérique du Nord. Jusqu' au début du XXe siècle, le Brésil reste la destination privilégiée, avec les États-Unis et l'Argentine, substitués après la Seconde Guerre mondiale par l'Europe et la France en particulier. Voir GodinHo, 1978.

4. PoINARD, 1988, p. 196, écrit du Portugal : « [...] la dispersion de communautés installées dans le monde porte témoignage de la vocation universaliste du pays [...] Et, on peut affirmer que c'est précisément la force de ce sentiment identitaire indiscuté [l'émigration en étant posée comme intrinsèque à la lusitanité, conforte l'appartenance identitaire des émigrés], allié au savoir-faire populaire migratoire, transmis par la famille et la communauté villageoise qui explique la rapidité avec laquelle les Portugais s'insèrent dans les sociétés d'accueil. »

5. Bonvalet et Villanova, 1999, p. 226. 
uniquement sur les dimensions économiques, politiques et culturelles de l'espace communautaire européen ${ }^{6}$, mais aussi sur la dimension identitaire.

La spécificité des liens symboliques, matériels, individuels et collectifs maintenus par des jeunes Portugais et d'ascendance portugaise de France avec le pays d'origine est ici examinée. Quelles raisons conduisent ces enfants de migrants, issus d'une population installée en France depuis les années 1960 et caractérisée comme étant bien insérée socialement ${ }^{7}$, à affirmer leur origine portugaise, perpétuer une pratique de «va-et-vient » entre les deux pays de référence et pour certains à s'investir dans le « retour » ? Les enfants de migrants construisent leurs identités à partir d'un ensemble de traits culturels spécifiques à chacune des deux cultures de référence, diversement manipulés en fonction d'interactions sociales, d'histoires individuelles et familiales. Les pratiques de circulation des enfants de migrants portugais à des fins estudiantines, professionnelles et familiales s'inscrivent dans une histoire collective de la migration. Illustrent-elles une gestion assumée d'une appartenance à deux espaces nationaux et culturels dans le contexte européen ? Existe-t-il un agencement de pratiques culturelles en fonction des deux espaces de circulation: langue, codes symboliques, capacités professionnelles...?

Au Portugal, la guerre coloniale et les conséquences sociales et économiques de l'Estado novo de Salazar, mais aussi l'appel de main-d'œuvre en provenance de l'Europe occidentale transforment une émigration structurelle en une émigration massive dans les années 1960 et 1970 en direction de la France principalement, pour la moitié de façon clandestine ${ }^{8}$. Il s'agit initialement d'un flux masculin provenant de régions pauvres, fondé sur un projet migratoire temporaire en vue d'améliorer les conditions de vie. Le regroupement familial permis par la loi française après la fermeture des frontières en 1973 a amplifié la dimension familiale de ce processus. Les immigrés portugais installés en France sont originaires d'un pays essentiellement rural, aux moyens de communication réduits et au taux d'analphabétisme élevé au moment de l'émigration. Ils proviennent le plus souvent des milieux paysans du nord et du centre du Portugal.

6. Libre circulation des travailleurs, équivalence des diplômes, programmes d'échanges interuniversitaires, citoyenneté européenne...

7. LEANDRO, 1995. L'auteur distingue les notions d'intégration et d'insertion sociale. Dans l'imaginaire collectif français, les Portugais sont caractérisés comme étant bien intégrés. Selon Leandro, les Portugais manifestent le désir d'adhérer à un processus d'insertion sociale, conservant leur identité culturelle. Selon PoINARD, 1988, p. 188 : «S'ils donnent l'impression d'être de "bons émigrés" qui ne créent pas de problèmes, ce n'est pas par la grâce d'une prétendue proximité culturelle, ou parce qu'ils sont "mûrs" pour l'assimilation, mais au contraire parce que la force de leur conscience identitaire leur permet de se plier aux règles de la société d'accueil sans avoir le sentiment de se nier.»

8. SERrÃo, 1982, p. 63-69. GodINHO, 1978, p. 12 : «[...] depuis toujours l'émigration clandestine, ou du moins non enregistrée, a été extrêmement importante. [...] nous la plaçons entre $1 / 4$ et $1 / 3$, le rapport variant selon les conjonctures. » 
De cette vie rurale, ils ont transporté un ensemble de valeurs, de façons de vivre, de penser et de croyances microlocales qu'ils ont cherché à perpétuer et à transmettre, car l'émigration était envisagée comme temporaire. À travers le cadre familial et associatif - il s'agit du plus vaste mouvement associatif communautaire de l'histoire de l'immigration en France ${ }^{9}$ - mais aussi par le biais de retours annuels, les familles portugaises ont perpétué des pratiques culturelles sur lesquelles reposent les sentiments d'appartenance à une identité collective.

Les pratiques de circulation observées chez des jeunes d'ascendance portugaise et les représentations des pays de référence qui les fondent émergent dans un contexte international en mutation. Les transformations structurelles qu'a connues la société portugaise depuis l'émigration parentale - amorcées dans les années 1960 (entrée du Portugal dans l'EFTA ${ }^{10}$ ), intensifiées par la révolution des œillets (1974), par l'adhésion du Portugal au Marché commun (1986) et son entrée dans la zone Euro en 1998 - conduisent d'une part, à une modification de l'image du Portugal et par conséquent de la représentation collective de l'origine portugaise, et d'autre part, à un décalage entre le pays réel et le pays affectif transmis par les parents. Il convient par ailleurs d'ajouter que, depuis les années 1980, a été observé chez les enfants de Portugais un allongement de la scolarité ${ }^{11}$ qui conduit à une mobilité socioprofessionnelle. Ces réalités posent notamment la question de la fidélité des générations issues de cette migration à une mémoire collective transmise.

\section{LA DOUBLE APPARTENANCE CULTURELLE : DISCOURS ET PRATIQUES ${ }^{12}$}

Éprouver le sentiment d'avoir deux cultures de référence et deux pays d'appartenance conduit un grand nombre de jeunes à s'interroger sur leur identité,

9. CORDEIRO, 1989, p. 27.

10. Accord signé en 1959 au traité de Stockholm entre le Portugal, l'Angleterre, la Suisse, l'Autriche et les trois pays scandinaves, créant une zone de libre-échange entre ces pays.

11. Portugal Branco, Ribeiro et Villanova, 1997, p. 73.

12. Les matériaux présentés dans cet article résultent de quatre années de recherches ethnographiques menées entre 1998 et 2001 en France (Région parisienne, Auvergne) et au Portugal (Lisbonne, Porto, régions d'émigration vers la France), auprès d'une cinquantaine d'enfants et de petits-enfants de migrants portugais, scolarisés dans la société française et pour plus de la moitié d'entre eux nés en France. En 1990, la moitié de l'effectif des 15-29 ans était née en France, voir Portugal BRANCO, 1996. En 1999, l'INSEE a recensé 553663 Portugais en France (645 578 en 1990), parmi lesquels les 15-29 ans représentent $15 \%$, auxquels viennent s'ajouter 235074 binationaux ou «naturalisés » - voir Portugal BRANCO, 2001 -, les 15-29 ans représentant 36,5\% de l'effectif. Les données exploitées ici sont celles recueillies dans des espaces collectifs, auprès de jeunes cherchant à valoriser et à affirmer leur origine portugaise : associations de «lusodescendants », cours de langue portugaise, rencontres internationales. 
et engendre souvent un certain malaise : celui « d'être entre deux pays », « d'être un étranger en France et au Portugal », celui d'une « perte identitaire ».

En juillet 1999, la Coordination des collectivités portugaises de France $(\mathrm{CCPF})^{13}$ organisa à Olhão (Portugal) la deuxième « Rencontre européenne de lusodescendants ». Plus de quatre-vingts jeunes Portugais et d'ascendance portugaise en provenance du Royaume-Uni, d'Allemagne, du Luxembourg, des Pays-Bas, de Suisse, de France et du Portugal, issus d'espaces associatifs portugais, ont été réunis autour d'un axe de réflexion : « la double culture ». Cet événement prolongeait une première rencontre organisée à Aveiro (Portugal), ainsi que trois années de «Rencontres régionales de jeunes d'origine portugaise » organisées par la CCPF dans différentes villes de France, en collaboration avec les associations portugaises locales. Partant du constat que, « de plus en plus, les jeunes Français d'origine portugaise affirment leur "portugalité” » et découvrent leur « double appartenance culturelle », illustrant leur volonté de « casser cette tradition de l'invisibilité de la communauté portugaise ${ }^{14}$, les responsables de ce collectif d'associations, tourné vers le développement du réseau associatif portugais et la promotion de la culture portugaise en France ainsi que vers l'insertion dans l'espace social français, ont orienté leur action auprès de la jeunesse autour de la question de la « double appartenance culturelle ${ }^{15}$ ». Un des ateliers de réflexion, organisés dans le cadre estival de cette rencontre, l'a été autour des questions : «Que signifie être lusodescendant?», «être portugais ou fils de Portugais dans le pays de résidence ? ».

Précisons que le terme «lusodescendant» apparaît, dans les travaux sur l'émigration portugaise, au milieu des années $1980^{16}$; il est notamment repris par la sociologue portugaise Maria Engrácia Leandro afin de dépasser les stéréotypes et les fantasmes concernant la dénomination des jeunes liés aux migrations internationales ${ }^{17}$. Les institutions portugaises (au Portugal et à l'étranger) ainsi que certaines associations liées au Portugal et à sa culture l'utilisent également. Étymologiquement, le terme « luso » est défini en tant que signifiant du Portugal - à la langue portugaise notamment : la lusophonie. Le terme « lusodescendant » devrait par conséquent désigner tous les Portugais. Or, ce terme désigne en fait « exclusivement» les descendants d'émigrés portugais résidant à travers le monde, possédant une seconde culture de référence à partir de laquelle ils construisent leurs identités : il s'agit dès lors

13. Un des deux collectifs nationaux d'associations portugaises en France. La CCPF regroupe environ 180 associations.

14. Brochure CCPF : « Jeunes d'origine portugaise, la double appartenance culturelle », Aubervilliers, s. d.

15. Ou encore : «l'identité nouvelle des "lusodescendants"».

16. ROCHA-TRINDADE, 1986.

17. LEANDRO, 2000, p. 6-7. 
d'un syncrétisme dépassant l'identité portugaise. Cette dénomination renvoie au contexte migratoire et permet de désigner l'ensemble des descendants de Portugais expatriés et leur héritage commun. Toutefois, contrairement au continent américain où l'on parle de «Luso-Brasileiros », de «Luso-Canadianos », l'utilisation du terme en Europe - « lusodescendant » - tend à effacer les réalités culturelles et nationales diverses auxquelles sont confrontés quotidiennement les jeunes d'origine portugaise de France, d'Allemagne, de Suisse... Or, Marie-Claude Muñoz se pose la question de savoir si cette «démarche ne manifeste [...] pas un refus d'assignation identitaire et une demande de reconnaissance de la dualité de leurs appartenances [... si elle] ne traduit pas la pratique sociale des acteurs qui circulent dans des espaces culturels différents ${ }^{18} \gg$. Peut-on voir dans cet usage européen du terme un lien entre la proximité avec le pays d'origine, proximité qui engendrerait des pratiques spécifiques?

L'apparition dans le mouvement associatif et l'appropriation du terme par des jeunes Portugais et d'ascendance portugaise illustrent une évolution dans la représentation de soi. Être «lusodescendant» ne renvoie pas au fait d'appartenir à une lignée familiale, la dimension généalogique ne semblant pas être ici privilégiée. Le terme renvoie à une origine nationale et/ou culturelle, mais aussi sociale et est associé à la notion de choix, celui d'assumer ou non ses origines. Il s'agit pour certains d'un terme valorisant dans le sens où Leandro l'utilise : il illustre le fait « d'être fils d'émigrants portugais, sans être soi-même un émigrant ». Sous ce même aspect, il rentre dans une logique de communication pour l'association Cap Magellan : «c'est une marque » qui ne renvoie pas à des stéréotypes ${ }^{19}$. Si pour certains jeunes ce terme « donne un nom à une situation », celle «d'être entre deux pays », pour d'autres jeunes qui en font usage, il permet de répondre positivement à un dilemme qui serait celui d'avoir à choisir entre deux cultures. Pour ceux-là, puisque être un « lusodescendant » ce n'est pas être uniquement portugais, cette dénomination autorise une « construction personnelle », une « création culturelle nouvelle » à partir des réalités culturelles et nationales de chacun. «Ce n'est pas posséder deux cultures, mais construire sur la base de deux cultures, une culture nouvelle », résumera Helena à Olhão (25 ans, secrétaire, Suisse).

Il faut préciser, cependant, que le terme «lusodescendant » est utilisé de façon restreinte à l'intérieur de cadres spécifiques : par certaines associations ${ }^{20}$, lors des rencontres internationales organisées par l'État portugais, par des

18. MuÑOZ, 1999, p. 8.

19. Au cours de l'année 2001, l'Association a entamé une série de campagnes publicitaires intitulées «Cap Magellan, le Portugal sans clichés » en instrumentant les stéréotypes relatifs aux Portugais de France, dont une majorité de jeunes avoue avoir souffert. Notons que c'est à partir de 1993 que le terme « lusodescendant » est utilisé dans le magazine de l'Association.

20. Associations Cap Magellan : «Cap Mag, Journal des Lusodescendants » (Paris), Lusomundo (Lyon), Lusogay à sa création, la CCPF. 
universités portugaises et françaises (l'usage étant relatif aux cours de langue portugaise destinés aux enfants d'émigrés/immigrés). En dehors des espaces collectifs, lieux d'affirmation de l'origine et de la culture portugaises, les jeunes conçoivent cette dénomination comme « scientifique », « politiquement correcte », ou encore ignorent son existence ${ }^{21}$.

L'usage politique du terme renvoie à la définition de la nation élaborée par Benedict Anderson. La lusodescendance inscrit des individus, séparés par des réalités nationales, culturelles et par des histoires familiales, dans une filiation commune qui prolonge matériellement et symboliquement la nation portugaise (définie par sa population plus que par son territoire). Elle donne toute sa continuité et sa dimension cosmopolite à la notion de «Communautés portugaises »: une « communauté imaginaire et imaginée » ${ }^{22}$.

Alors que l'appartenance à deux cultures semble un fait plus ou moins établi, s'agissant de la nationalité un choix s'impose dans certains discours. En effet, chez les jeunes binationaux, une priorité est accordée à la nationalité portugaise, transmise par filiation : celle-ci apparaîtrait comme «supérieure » à

21. Ajoutons qu'il existe un aspect plus polémique du terme, «luso » renvoyant aux Lusitaniens (ou Lusitains), peuplade préromaine fixée dans un territoire correspondant à une fraction du Portugal actuel, dont les frontières demeurent inchangées depuis le $\mathrm{XII}^{\mathrm{e}}$ siècle, voir Portugal Branco, 1999, p. 29. L'anthropologie portugaise à son essor - fin du XIX ${ }^{\mathrm{e}}$ siècle est une « anthropologie de construction de la nation » associée à un discours aux prétentions « ethnogénéalogiques » sur le Portugal, faisant de la nation portugaise une « communauté ethnique de descendance », voir LEAL, 2000, p. 64. La discipline exhume pour ce faire les Lusitaniens, réutilisés par la suite par l'idéologie de l'État nouveau comme symbole du caractère commun et unique de la nation portugaise. Les Lusitaniens et la notion de « lusodescendance » renvoient pour certains - un exilé politique rencontré en France par exemple - au mythe de la pureté originelle, au culte de la lusitanité instrumenté sous la dictature.

22. ANDERSON, 1996. À la fonction économique de l'émigré portugais, dont les transferts d'épargne ont été décisifs pour la solvabilité de l'État (notamment après la révolution des (Eillets), vient s'ajouter une fonction symbolique. Dans les années 1980, les émigrés portugais deviennent les «Communautés portugaises », création en 1980 du «Conseil des communautés portugaises », un organe consultatif « ayant pour fonction de sensibiliser le gouvernement aux problèmes des émigrés » (propos de Manuela AGUIAR, ancien secrétaire d'État aux communautés portugaises ayant théorisé et mis en pratique la politique des Communautés), par ailleurs le Secretaria de Estado da Emigração devient le Secretaria de Estado da Emigração e das Comunidades Portuguesas ; en 1981, le code de la nationalité portugaise fondé sur le droit du sang instaure « une véritable loi du retour », voir POINARD, 1988, p. 198 : « [...] seul sera déchu de sa nationalité celui qui, faisant la preuve d'en avoir acquis une autre, établit une déclaration de rejet auprès des autorités portugaises compétentes » (art. 21, alinéa 3) ; droit à la participation aux élections législatives et plus récemment aux élections présidentielles (2001) ; promotion de la fête nationale du 10 juin Dia de Portugal e de Camões (célébration de la mort du poète portugais Luis de Camões 1524 ?-1580) devenu Dia de Raça (Jour de la race) sous Salazar, en Dia de Portugal, de Camões e das Comunidades; érection de monuments commémoratifs en l'honneur des émigrés... 
la française, acquise à la majorité ${ }^{23}$. Le sentiment d'appartenance nationale d'une majorité de jeunes d'ascendance portugaise est en effet fondé sur la parenté (restreinte) : «le lien du sang », comme certains aiment à l'exprimer. Cette appartenance renvoie plus concrètement à la région dans laquelle se situent les villages natals des parents : « Je suis Portugais de telle région. » Le Portugal est un pays caractérisé par une forte diversité régionale ${ }^{24}$. L'origine régionale des Portugais et des émigrés est de ce fait très prononcée, entrant dans le jeu des relations sociales. La représentation du Portugal chez les émigrés portugais et leurs enfants renvoie à des micropatries ${ }^{25}$ : au village d'origine, associé à la ville la plus proche et non à la totalité du pays, qui reste globalement inconnu.

L'appartenance nationale est un sentiment présent dans le discours et observable dans les pratiques de jeunes d'origine portugaise. Il se traduit par des conduites patriotiques, notamment affirmées dans un espace collectif communautaire, sportif, artistique et de festivité (discothèque « portugaise » en banlieue parisienne, associations...). Il est ainsi fréquent de voir le drapeau portugais exhibé de différentes manières dans cet espace, d'y entendre l'hymne national portugais ${ }^{26}$. Le sentiment national constitue, selon Gérard Noiriel, « une ressource identitaire latente que les individus mobilisent dans certaines circonstances, le plus souvent, le sport captant le sentiment de fierté nationale et la charge émotionnelle qui l'accompagne ${ }^{27} \gg$. Si les matchs de football ont depuis longtemps fourni l'occasion de mobiliser les sentiments d'appartenance nationale, celle-ci est depuis peu également utilisée dans d'autres contextes, comme dans le cas du groupe de rappeurs franco-portugais (La Harissa) lors de ses concerts (à Paris, à la Cigale, ou encore dans la discothèque portugaise la Costa do Sol à Villeneuve-Saint-Georges) et de soirées associatives : à travers le rap, «musique de ghetto et d'identité », le groupe revendique la reconnaissance de l'immigré portugais et de sa culture populaire, instrumentant la mémoire de l'émigration

23. Dans le cas des émigrés installés en France, un grand nombre de leurs enfants nés en France, mais par ailleurs enregistrés dans la zone consulaire de naissance, ont acquis la nationalité française à leur majorité (droit du sol français) en gardant la nationalité portugaise pour l'État portugais, voir PORTUGAL BRANCO, 2001.

24. MatToso, 1998, p. 10 : l'idée de l'unité culturelle et linguistique du Portugal constitue un présupposé.

25. Terme emprunté à RoCHA-TRINDADE, 1987, p. 727 : ainsi ont été réunies les conditions, aussi bien affectives que matérielles, au plan interne comme à celui de la relation externe, pour que chaque terre se présente, aux yeux de ceux qui y vivent comme à ceux des émigrants, comme leur petite mais unique patrie.

26. Le drapeau portugais illustrerait la vocation universelle du peuple portugais : la sphère armillaire, au centre, représentant l'univers et par conséquent consacrerait le rôle joué par le Portugal comme découvreur, voir LouRENÇO, 1997, p. 8. Ce symbole est généralement ignoré des jeunes.

27. NOIRIEL, 2001, p. 138. 
portugaise. À ce propos, pour le sociologue Sergio Lopes, le patriotisme « portugais » de ces jeunes est un indicateur d'intégration à la culture française : «D'une certaine façon, un bon nombre de revendications de "lusitanité" sont un pur produit du terroir français ${ }^{28}$. » Il est en effet intéressant d'observer que certains de ces jeunes, fréquentant des discothèques portugaises en France, se rendent au Portugal dans des discothèques où l'animation valorise leur origine « française » (Porto, Pombal). Les animateurs s'adressent à eux en français, diffusent de la chanson française et cherchent à connaître les villes dont ils sont originaires en France : le sociologue Jorge de Portugal Branco parle à ce propos de «perplexité identitaire ${ }^{29}$ ».

L'ambiguïté du positionnement identitaire des jeunes observés - se sentir français au Portugal et portugais en France - tient à la nature des projets migratoires familiaux tournés vers un retour perpétuellement ajourné, qui conduit notamment à une idéalisation de la vie dans le pays d'origine, pourtant loin de représenter le vécu des émigrés lors des retours annuels. Cette ambiguité met aussi en évidence le silence d'une mémoire familiale : mémoire de la migration composée de traumatismes et d'indicible. L'émigration prédispose en effet à l'enfouissement, mais aussi à la volonté d'oubli de ce qui fait souffrir ${ }^{30}:$ le voyage dans la clandestinité qui a parfois duré plusieurs semaines, la séparation d'avec la famille, d'avec la «terra», les bidonvilles... « Pour les descendants de la nation maritime qui avait conduit le navire Europe par les océans, cette descente à la cale européenne pourrait se comparer à une descente aux enfers », écrit Eduardo Lourenço ${ }^{31}$. Les parents deviennent dans certains récits de vie de véritables héros. Des traumatismes familiaux, mais aussi individuels existent, relatifs au vécu quotidien: les préjugés, les pratiques discriminatoires (et en particulier scolaires), les stéréotypes attachés à l'origine portugaise que l'enfant a cherché à dépasser de différentes manières.

À ce propos, l'étude de l'espace associatif est particulièrement intéressante, illustrant la volonté de jeunes Portugais et d'ascendance portugaise de construire une nouvelle image des Portugais et du Portugal en France ${ }^{32}$. Au cours des

28. LOPES, 1998, p. 72.

29. Séminaire de recherche Unité de recherches Migrations et société : «La communauté portugaise », université Paris VII, mars 2001.

30. Villanova, 1999, p. 23.

31. LOURENÇO, 1994, p. 212.

32. L'implication associative des jeunes d'origine portugaise est restreinte, mais l'espace est cependant utilisé pour y vivre collectivement de manière épisodique son appartenance culturelle, ainsi que comme espace matrimonial. La perpétuation de la pratique associative chez les jeunes d'origine portugaise est un sujet de préoccupation au sein de la communauté portugaise : en témoigne la thématique des Rencontres européennes de lusodescendants organisées annuellement par la Coordination des collectivités portugaises de France, depuis deux ans orientées sur l'implication associative des jeunes. 
années 1990, des jeunes ont cherché à s'impliquer dans les associations créées par leurs aînés, en accédant à des postes de dirigeants et en organisant de nouvelles activités : Comunidade Portuguesa de Formação Cultural Le Raincy e Arredores (cours de portugais, sorties culturelles, cinéma...), Agora à Argenteuil (danse moderne, informatique...), O Sol de Portugal à Bordeaux (accompagnement scolaire adressé aux différentes populations étrangères, informatique, «Luso contes »...). Des associations ont également été créées par des étudiants portugais et d'origine portugaise - Association des étudiants lusophones (Nancy, 1988), Cap Magellan (Paris, 1991), La Caravelle d'Orphée (Nantes, 1993), Lusomundo (Lyon, 1994), Lusogay (Paris, 1998), Le Cercle des poètes lusofones de Paris (1998), Accord'Art (Paris, 2000) - orientées vers une affirmation du Portugal à travers la mobilisation d'une « culture valorisée ${ }^{33}$ » et son affirmation au sein de la société française. Ces associations utilisent les nouvelles technologies pour communiquer (informatique, Internet), les médias français et portugais : journaux locaux (Le Parisien, Le Républicain lorrain...), journaux communautaires (Encontro, Lusitano, Opinão), radio communautaire (radio Alfa) et radios locales (à Lyon, à Bobigny), télévision régionale française (France 3) et internationale portugaise (RTPI). L'ouverture sur la société française reste cependant limitée. Le président de l'association Cap Magellan semble toutefois mettre en œuvre une « stratégie de placement et de conquête d'un statut à l'intérieur des deux collectivités nationales, d'origine et de résidence », selon les termes de René Gallissot ${ }^{34}$, comme l'illustre la présence de l'ambassadeur du Portugal, du secrétaire d'État portugais à l'enseignement supérieur ${ }^{35}$ ainsi que du maire de Paris, à « Tosmania ${ }^{36}$ » : fête commémorative organisée le 10 juin 2001, célébrant la fête nationale portugaise (dédiée aux Communautés portugaises). Une centaine de « lusodescendants » étaient réunis au Divan du Monde à Paris.

Dans ces espaces associatifs, il n'est plus question de «tradition » ni «d'authenticité », valeurs auxquelles la pratique folklorique (omniprésente dans les associations créées par leurs aînés) renvoie ${ }^{37}$. La priorité est désormais accordée au théâtre, à la poésie, au cinéma et à la musique en vogue au Portugal et plus largement dans l'espace lusophone (Afrique, Brésil). La maîtrise de la langue portugaise est par ailleurs valorisée notamment comme un atout

33. Caractérisée comme telle par rapport aux éléments de la culture portugaise qui leur ont été transmis par leurs parents.

34. Gallissot, 1993, p. 12.

35. Ce dernier a loué le « cosmopolitisme portugais, l'ouverture du pays sur le monde et sa présence en France ».

36. «Tos » étant une des dénominations, initialement à caractère péjoratif, des Portugais en France. Voir aussi MuÑoz, 1999.

37. Voir Irène Dos SANTOS, « Ethnographie d'un rancho folklorique d'immigrés portugais. Mise en scène de la tradition comme support de l'expression d'une identité culturelle », mémoire de D.E.A., Paris, École des hautes études en sciences sociales, 1998. 
professionnel, une plus-value sur le marché de l'emploi ${ }^{38}$. Ces associations semblent constituer des lieux d'épanouissement personnel, qui offrent à leurs membres la possibilité de se construire une nouvelle image de leur origine portugaise, puisant dans un capital et un héritage élargi, plutôt qu' uniquement familial ${ }^{39}$. Cet investissement des « lusodescendants » dans un capital culturel portugais autre que celui transmis par leurs parents devra par ailleurs susciter une réflexion sur ses répercussions dans les relations intergénérationnelles ${ }^{40}$.

L'histoire du peuple portugais constitue un capital symbolique national sur lequel se fonde l'identité nationale portugaise ${ }^{41}$. Le thème des découvertes maritimes apparaît comme métaphore positive de l'émigration dans l'espace associatif (association « Cap Magellan ${ }^{42}$ », chanson du groupe La Harissa intitulée « Conquistador ${ }^{43}{ }$...). Il est également omniprésent dans les discours des politiques adressés aux émigrés et à leurs descendants. Les politiques et idéologies coloniales successives du Portugal - inclusivement celles liées à l'émigration - illustrent « la constante (ré)invention du rôle légendaire accompli par les navigateurs portugais à l'époque des grandes explorations maritimes ${ }^{44} \gg$. Par ailleurs, des éléments de l'identité nationale portugaise fonctionnent également comme ressources identitaires : la saudade ${ }^{45}$, instrumentée sous la dictature en tant que trait distinctif du caractère portugais, et le fado, transformé en archétype de la chanson nationale portugaise ${ }^{46}$. De plus, l'émigration, dont sont issus les jeunes Portugais et d'ascendance portugaise de France étudiés, a eu lieu sous une dictature dont l'idéologie visait la mise en évidence d'un patrimoine populaire légitimant le maintien de valeurs traditionnelles, dans un contexte d'exaltation de l'identité et de la culture nationales ${ }^{47}$. Ce dernier aspect

38. L'association Cap Magellan possède un département Stages et emplois, dont l'objectif est de faire le lien entre les entreprises françaises et portugaises et les « lusodescendants » en quête d'emploi.

39. LA BARRE, 1997.

40. I. Dos SAnTos, «Mémoires collectives dans la migration portugaise », actes de la rencontre Epoca «Les Portugais et le Portugal en France au XX $\mathrm{X}^{\mathrm{e}}$ siècle », Nanterre, Bibliothèque de documentation internationale contemporaine, 8 déc. 2001, Archives et histoire des relations internationales, Cahiers de recherches, 2003, p. 71-82.

41. Mattoso, 1998.

42. Le champ lexical da la navigation est très largement exploité dans la revue mensuelle de l'association : « l'équipage [...] à la barre [...] », « notre voyage se poursuit, en des mers plus calmes $[\ldots] »$.

43. « Les plus grands navigateurs que la planète ait pu connaître sont de retour. » Notons, par ailleurs, que le terme ne renvoie pas aux « découvertes » portugaises mais aux «conquêtes » espagnoles.

44. Feldman-Bianco, 1995, p. 90.

45. Désir d'un bien dont on est privé, regret de l'absence (Dicionários Bertrand). Voir aussi LOURENÇO, 1997.

46. LEAL, 1999.

47. Melo, 2001 ; CunHa, 2001. 
est important au niveau des répercussions sur les représentations du Portugal et des éléments de l'identité portugaise transportées et transmises par l'émigré portugais (le folklore étant l'un des aspects manifestes). L'histoire « circule [dans l'émigration] sous une version le plus souvent réduite et stéréotypée ${ }^{48} »$ : les Découvertes, le roi Sébastien, le poète Luis de Camões, la révolution des Eillets. Si l'on peut y voir le rôle joué par l'enseignement de la langue et de la civilisation portugaises auprès des enfants de Portugais, il convient également d'analyser ces réappropriations comme illustrant le choix de traits culturels portugais opérant dans la société française et pour elle reconnus : le fado et le vin de Porto (non consommés avant l'émigration), le navigateur Magellan (davantage connu en France que Vasco de Gama, symbole des découvertes au Portugal)... Les recherches généalogiques entreprises par des jeunes d'ascendance portugaise auprès de l'ambassade du Portugal à Paris vont dans le même sens. L'article partitif n'existant pas dans la langue portugaise, les « de », « da(s) », « do(s) » ne sont en aucun cas des particules et ne signifient nullement une origine noble, comme c'est le cas en France. La quête d'une reconnaissance sociale pour certains jeunes passe ici aussi par l'appropriation de codes symboliques français.

L'étude de la manipulation des prénoms illustre l'agencement de pratiques identitaires en fonction d'espace de circulation. Les jeunes femmes, notamment, adaptent l'orthographe et la prononciation de leurs prénoms - la forme française ou portugaise : Cristina/Christine, Ana Sofia/Anne-Sophie, Isabel/Isabelle en fonction de contextes sociaux et nationaux ${ }^{49}$. Si le prénom est un marqueur familial $^{50}$ - transmission intergénérationnelle des prénoms -, il devient également un marqueur culturel avec lequel ces jeunes jouent : être semblable (utilisation du prénom « portugais » au Portugal, en famille, utilisation du prénom français à l'école) ou être différent (utilisation du prénom « portugais » en France, afin de s'affirmer en tant que Portugais).

L'usage de la langue portugaise répond à ces mêmes enjeux. Elle est transmise et perpétuée dans l'espace familial et également enseignée par le biais associatif. Des élèves (14-17 ans) rencontrés dans cet espace s'investissent de façon majoritaire dans l'apprentissage de la langue en vue de « retourner » au Portugal : « Nous avons tous la même histoire [...] si vous posez la question, tous on vous dira qu'on veut repartir » (Association culturelle d'études portugaises, Paris). Dans certaines familles, la pratique de la langue portugaise est strictement réservée à l'espace domestique, illustration des «stratégies d'invisibilité » adoptées à l'extérieur afin de ne pas être vu comme un immigré, de visibilité à

48. ORIOL, 1985, p. 209.

49. Une manipulation à laquelle les incohérences bureaucratiques les incitent largement.

50. ZONABEND, 1980. 
l'intérieur de la maison, espace d'expression de la culture d'origine ${ }^{51}$. Dans certaines familles, on observe des échanges dans les deux langues : «Mes parents me parlent en portugais, je réponds en français », lorsque la langue portugaise n'est pas suffisamment maîtrisée par les jeunes, ou bien lorsque ces derniers ne s'y identifient pas. Les pratiques d'alternance des deux langues sont fréquentes, elles permettent aux membres d'une même fratrie de vivre des positions différenciées vis-à-vis des deux langues et des deux cultures : « $\mathrm{Au}$ cœur de ces pratiques, se tissent la mémoire familiale et l'inscription symbolique du sujet dans un espace libéré des frontières géographiques ${ }^{52}$. » Lors des retours annuels au Portugal, l'usage du portugais autorise une plus grande proximité avec la famille et symbolise la continuité des liens généalogiques. Ne pas parler portugais peut réduire considérablement les échanges avec les grands-parents qui jouent un rôle essentiel dans la transmission de la mémoire familiale. Il est toutefois important de préciser que lorsque les grands-parents ont eux-mêmes émigré, le français devient la langue par le biais de laquelle la transmission s'effectue, même au Portugal. Par ailleurs, une pratique souvent approximative du portugais (les néologismes franco-portugais sont fréquents ${ }^{53}$ et les codes symboliques complexes ${ }^{54}$ ) et connotée socialement: "Mes parents m'ont transmis une langue vulgaire » (Tony, étudiant, 22 ans, Paris), ainsi que le recours au français dans des lieux publics, stigmatisent l'émigré et ses descendants au sein de la société portugaise.

Le français et le portugais sont deux langues mobilisées dans les parcours professionnels : en fonction du pays, le français et le portugais sont valorisés dans l'enseignement, dans le bancaire (agences portugaises en France par exemple), dans les multinationales et le tourisme... Le choix d'un cursus universitaire qui mobilise le portugais est fréquent ${ }^{55}$. De même, lors des retours au Portugal, le choix d'un cursus universitaire qui mobilise les langues est souvent

51. Stratégie observée par LEANDRO, 1995, p. 326, à Paris (16 ${ }^{\mathrm{e}}$ arrondissement) et non en banlieue parisienne : «[...] imposée par le contexte social local qui accepte difficilement les démonstrations de présence des groupes minoritaires sociaux ou ethniques hors du travail et des actes de culte du dimanche. »

52. Villanova, 1986, p. 179.

53. Exemples : arriver-chegar : arrivar; bouteille-garrafa : botelha; maladie-doença: maladie ..., in Maria Engrácia LEANDRO, « As interconexões da língua portuguesa em situação imigratória », polycopié, Braga, s. d.

54. Exemple de l'utilisation presque systématique du «vocêe pour le vouvoiement (par similitude avec le vous), alors qu' au Portugal ce terme introduit traditionnellement une dimension hiérarchique.

55. Sur 2221 étudiants de nationalité portugaise recensés pour l'année scolaire 2000/2001 (les binationaux sont recensés par l'INSEE en tant que Français), 527 sont en langues : il s'agit de l'effectif le plus important, bien qu'en légère baisse depuis 1980, voir PORTUGAL BRANCO, 2001. 
préféré56. Ces pratiques fondées sur l'affectif conduisent à des échecs et à des déceptions dus, soit à une maîtrise insuffisante des connaissances grammaticales et littéraires exigées pour l'enseignement du français au Portugal ${ }^{57}$, soit à une prise de conscience tardive des limites professionnelles des études de portugais quand elles ne sont pas associées à une autre compétence.

La lusophonie effective et plus encore le choix d'un conjoint portugais ou d'ascendance portugaise sont des marqueurs d'identité pour les jeunes enquêtés dans ce cadre spécifique : " J'aimerais que ce soit un enfant d'émigrés comme moi pour qu'il puisse me comprendre » (Paula, 25 ans, étudiante, Porto). Les associations et leurs soirées constituent des lieux de rencontre entre jeunes d'origine portugaise. Le choix du conjoint influence le projet de vie ou est influencé par lui. L'endogamie semble dans certains cas représenter un idéal, l'exogamie représentant symboliquement un obstacle au «retour» vers le pays d'origine, notamment dans la pratique de ceux qui rêvent de s'installer au Portugal (Paula, David - 23 ans, ouvrier en bâtiment, Région parisienne -, Daniel - 27 ans, ingénieur télécommunications, Paris -, ...).

LE POIDS DU PROJET MIGRATOIRE FAMILIAL

«Ser lusodescendente significa ter Portugal sempre como país da escolha para fazermos a nossa vida ${ }^{58}$.»

Le verbe «Émigrer » est défini par Émile Littré comme « le fait de quitter, de façon contrainte ou non, sa patrie pour des raisons économiques ou idéologiques. Ce terme s'applique à ceux qui sont partis avec l'intention de revenir ; les autres étant devenus des étrangers ou des colons ». L'idée d'émigration est constituée par l'idée sous-jacente d'un retour presque toujours présente dans l'imaginaire de ceux qui sont restés comme de ceux ayant quitté le Portugal, mais n'est pas concrétisée ${ }^{59}$.

56. Selon les chiffres du ministère de l'Éducation portugais (direction générale de l'Enseignement supérieur) relatifs aux candidats d'origine portugaise («Contingente de Emigrantes ») au concours national d'entrée dans l'enseignement supérieur pour l'année 1996/1997, $30 \%$ des candidatures concernent la section «lettres, secrétariat, traduction, interprétariat ».

57. Qui a par ailleurs perdu beaucoup d'importance dans la société portugaise, remplacé par l'anglais. Le français tend par conséquent à constituer de moins en moins un atout professionnel pour les «lusodescendants » de « retour».

58. «Être lusodescendant signifie avoir toujours le Portugal comme le pays choisi pour faire notre vie » (Olhão).

59. Monteiro, 1994. 
Concernant les Portugais ayant émigré vers le nord de l'Europe dans les années 1960, 51280 retours ont été recensés entre 1977 et 1981 et 32240 entre 1997 et $1998^{60}$. Les projections de l'INSEE estiment entre 15000 et 20000 le nombre de retours annuels des Portugais de France ${ }^{61}$. Cependant, selon Policarpo Lopes, « le retour en masse prévu ne s'est pas réalisé, car la circulation des émigrés portugais correspond à une nouvelle stratégie de "migration-circulation" qui s'insère dans la globalisation et est facilitée par les droits inhérents à la citoyenneté européenne ${ }^{62}$ ».

La notion du retour est sublimée dans la culture portugaise, comme l'illustre le sébastianisme (croyance résiduelle selon laquelle le roi Sébastien ne serait pas mort à la bataille d'Alcácer Quibir en 1578 et reviendrait pour restaurer l'unité du Portugal et fonder un empire universel, le cinquième empire ${ }^{63}$ ), auquel se réfèrent quelques jeunes interrogés pour légitimer leur désir de « retour ». Par ailleurs, le statut reconnu aux émigrés portugais et à leurs descendants à travers la loi de 1981 sur la nationalité constitue une véritable « loi du retour»: il reconnaît « le droit à l'intégration dans une autre société tout en garantissant le droit imprescriptible à revenir dans la mère-patrie ${ }^{64} »$.

Le retour aux « racines », à la « terra », au « pays d'origine » est omniprésent dans le discours et les pratiques des familles portugaises rencontrées en Région parisienne ${ }^{65}$. La vie des migrants portugais est jalonnée par des retours temporaires - pratique de « va-et-vient ${ }^{66}$ »- ou définitifs, programmés ou rêvés : à l'occasion des fêtes religieuses, des vacances d'été, au moment de la retraite ou, après décès, le retour symbolique sur la «terra ». Les contacts maintenus avec la communauté villageoise se matérialisent à l'occasion du voyage annuel qui prend l'allure d'un rite principalement effectué en août. Ce retour a généralement lieu dans un des villages ou une des villes d'origine, ou à proximité de celui-ci ou de celle-ci, en fonction du lieu choisi pour la construction de la maison le cas échéant. La localisation de la maison est l'expression des affinités familiales, mais surtout d'une tradition d'uxorilocalité et d'uxorivicinalité,

60. RATO, 2001, p. 165.

61. Jorge Portugal Branco, «Escolarização dos Portugueses em França na década de 80 », document polycopié, Paris, Ambassade du Portugal, déc. 1992.

62. Policarpo Lopes, Portugal, holograma da mobilidade humana, Lisbonne, Editora Rei dos Livros, 1999, cité in RATO, 2001, p. 165.

63. Le sébastianisme évolua vers le patriotisme et aboutit à la restauration de la couronne portugaise (1640), après quelques décennies de domination espagnole, voir MARQUES, 1998, p. 230. Voir aussi VALENSI, 1992.

64. PoINARD, 1988, p. 198.

65. Lors d'un terrain d'enquête en Auvergne, nous avons pu observer, d'une part, que le projet du retour était moins central dans les préoccupations des familles portugaises et, d'autre part, que le phénomène de double résidence y était moins marqué.

66. Charbit, Hily et Poinard, 1997. 
notamment dans le nord du Portugal ${ }^{67}$. La construction d'une maison s'inscrit dans le processus migratoire : «La lutte contre la précarité du logement, rarement évoquée spontanément par les immigrés eux-mêmes, n'apparaît pas, de prime abord, dans les raisons d'émigrer [...] Pourtant, il semble bien que le désir de maison soit inscrit dans le projet de migration ${ }^{68}$. $\gg$ Si la maison construite au Portugal demeure prioritaire dans un grand nombre de projets immobiliers d'immigrés portugais, celle-ci n'exclut pas le phénomène de double résidence engendrant la pratique de l'alternance résidentielle ${ }^{69}$. La maison construite au Portugal constitue aussi un lieu de retrouvailles pour les membres d'une famille dispersée dans l'émigration : David installé à Paris y retrouve ses parents et son frère qui vivent durant l'année à Nantes ; les membres de la famille M. dispersés entre la France (parents et fils aîné) et le Portugal (fils cadet) s'y réunissent. «La double résidence constitue l'espace mythique de la cohésion familiale et de l'affirmation sociale [...]», écrit Carolina Leite ${ }^{70}$. Les retours temporaires permettent de garder un lien continu dans un lieu occupé par la mémoire familiale et avec un Portugal « authentique » : les soirées passées au coin du feu dans la vieille maison en pierre des grands-parents, le travail des champs, la fête où l'on tue le cochon... La notion de «terra » renvoie, selon Paulo, au « pays [...] aux hommes qui y vivent, aux terrains hérités ».

Les fêtes familiales et villageoises, religieuses et profanes sont des espaces de retrouvailles entre migrants dispersés et villageois résidents : fêtes des émigrants (messe, procession, folklore, tournois de football entre équipes locales et d'émigrants, bals...), mariages, anniversaires de mariage et baptêmes ont lieu au cours de ce mois ${ }^{71}$. De nombreux villages ont déplacé une grande partie de leurs activités festives au mois d'août. « La célébration au pays des baptêmes et des mariages, qui rassemblent toute la parenté, traduit le besoin de s'inscrire dans une lignée et l'affirmation d'une fidélité à la terre d'origine », écrit Muñoz ${ }^{72}$. Les enfants d'émigrés se marient fréquemment au village d'origine des parents de la mariée, quand les deux époux sont d'ascendance portugaise ; il s'agit du mariage religieux, le mariage civil étant préalablement célébré en France, à la mairie ou encore au consulat ${ }^{73}$. Les célébrations de ces rites de

67. CALlier-Boisvert, 1999.

68. Bonvalet et Villanova, 1999, p. 222.

69. Bonvalet et Villanova, 1999, p. 246. Voir aussi Villanova, Leite et Raposo, 1994.

70. LeITE, 1999, p. 308.

71. Charbit, Hill et Poinard, 1997, p. 47, font remarquer que : «C'est précisément le décalage entre la date de naissance et celle du baptême qui fait sens et révèle cette fonction centrale de rassemblement joué par le village. » Dans certaines familles portugaises immigrées, nous avons observé l'importance donnée à l'éducation religieuse des enfants (catéchisme portugais ou français) et notamment aux sacrements d'initiation chrétienne (baptême, première communion et confirmation) qui autorisent le mariage religieux au Portugal.

72. MuÑOZ, 1998, p. 42.

73. Nous avons également pu observer chez des couples mixtes des célébrations religieuses au Portugal. 
passage prennent l'allure de retrouvailles entre Portugais expatriés, migrants intérieurs et résidents.

Si le retour définitif en masse des émigrés portugais ne semble pas se vérifier, des retours effectifs, autres que dans le cadre des vacances et de la retraite, sont cependant enregistrés :

«La communauté française du nord du Portugal, qui est en augmentation régulière depuis le début des années 1990 pour atteindre aujourd'hui plus de 3600 personnes, s'est considérablement transformée au cours des dernières années : il y a moins de cadres expatriés, mais plus de Français dans toutes les catégories socioprofessionnelles et en particulier des français d'origine portugaise [...] beaucoup, à la deuxième génération en particulier, ont acquis la nationalité française et ont décidé de revenir ou de venir s'installer au Portugal ${ }^{74}$. »

À ce propos, notre rencontre avec Christelle (étudiante, 22 ans, Région parisienne) qui a choisi d'écrire son mémoire d'anglais sur : «The Portuguese Dream » est intéressante. Ce travail est fondé sur sa propre expérience de vie. Elle y relate le départ vers le Portugal «d'une grande partie de ses copains, cousins et de son frère, avec le baccalauréat en poche pour y faire carrière », confiant par ailleurs que : « [elle] le dénonce [...] ils n'ont aucune raison valable pour m'expliquer leur désir de repartir. »

Les projets de « retour» des jeunes d'origine portugaise - terme d'usage souvent impropre puisque la moitié d'entre eux n'est pas née au Portugal, elle n'y retourne donc pas mais y part - sont complexes quant à leurs motivations. Les candidats au « retour » sont souvent attirés par une vie meilleure (la sociabilité villageoise est fréquemment mise en avant), par une expérience professionnelle (le taux de chômage étant inférieur à celui de la France et l'idée encore répandue que les diplômes français y sont valorisés), ou encore estudiantine (dans le cadre du programme européen d'échange interuniversitaire Erasmus ; il existe par ailleurs un quota de places $(7 \%)$ réservées aux « émigrants » dans l'enseignement supérieur portugais). Paulo inscrit son désir d'aller vivre et travailler au Portugal dans le projet migratoire familial : il confie avoir «été conditionné au retour ». Pour certains jeunes, la raison du départ réside dans le désir de mieux connaître le Portugal, afin de faire "un choix [qui s'avère] nécessaire » entre les deux pays ${ }^{75}$. Pour d'autres, il est guidé par le sentiment de

74. Consulat général de France à Porto, 1999, « Regard sur la présence française au nord du Portugal », Livret du Français à l'étranger, Direction des Français à l'étranger. Le journal Libération : «Retours aux sources », 9 avril 2001, et l'hebdomadaire Le Point : « Portugal, le difficile retour », juillet 2000, « Portugais de France. Le fado du retour », 10 août 2001, ont publié dans leurs rubriques «emploi » et «monde» des articles sur «le retour des enfants d'immigrés portugais ».

75. « Je dois faire un choix. Entre deux [pays] tu n'es pas bien [...] tu ne peux pas vivre avec les deux en même temps » (Paula). 
se sentir davantage « chez soi » au Portugal. Le fait que le climat y soit amène et « la vie plus agréable », renvoyant aux pratiques de sociabilité expérimentées au cours des vacances, est fréquemment évoqué. Il est par ailleurs intéressant d'observer que lors des expériences individuelles au Portugal, les jeunes se rendent en fin de semaine dans le village d'origine où ils s'installent « chez eux », à savoir dans la maison construite par leurs parents : «Forcés par les difficultés croissantes d'insertion professionnelle en France, cette maison constitue un ancrage à un territoire qui risque, à tout moment, de se constituer comme alternative de vie », écrit Leite à propos des jeunes d'ascendance portugaise ${ }^{76}$. Ils gardent des liens étroits avec la famille d'origine, notamment à travers les repas partagés chez grands-parents, tantes et cousins ${ }^{77}$. Une expérience de vie au Portugal permet souvent, à ceux pour qui l'idée de retour est omniprésente et la vie dans le pays d'origine de leurs parents idéalisée, une prise de conscience de leur spécificité culturelle, de sa richesse, ainsi que de la réalité portugaise.

Si nous pouvons distinguer les retours temporaires des retours définitifs tels qu'ils sont eux-mêmes projetés par les candidats, seule la nature de l'expérience effectivement tentée, décidera d'une installation définitive ou non de l'intéressé. Un premier retour temporaire peut servir de tremplin à un retour conçu comme définitif : une expérience Erasmus réussie peut susciter l'envie d'y poursuivre une expérience professionnelle. Elle peut aussi faire prendre conscience des décalages entre les attentes personnelles et la réalité, impossibles à percevoir lors des vacances :

«Les gens sont conservateurs [...] chez le boulanger ou le photographe, quand on me demandait de répéter ma phrase, c'était terrible [...] les gens se méfient de nous [...] un jour je me suis dit : il faut que j'aime la pluie pour être bien ici [...] alors j'ai commencé à aimer la pluie [...] finalement, si eux peuvent vivre ici moi aussi » (Sophie, salarié associatif, 25 ans, Paris) ${ }^{78}$.

Un des motifs de retour évoqués est aussi celui de ne pas faire échouer le projet parental : «Suis-je capable de vivre ici au Portugal ? [...] mon choix aura des conséquences sur la vie de mes parents, sur le choix du pays de la retraite » (Paula) ; «Je veux que ma mère se casse, elle est malheureuse ici, si mon frère revient ils vont rester » (Paulo). Ce dernier propos illustre le cas d'une famille portugaise installée à Paris, dont le fils cadet est parti en 1997 « plein d'illusions »- suivre des études de médecine au Portugal. Il rejoignait

76. LEITE, 1999, p. 312.

77. Le choix de l'université est souvent effectué en fonction du lieu de vie de la famille et de l'emplacement de la maison des parents.

78. Après une expérience Erasmus d'un an à Braga (région d'origine des parents), Sophie a travaillé un an à Paris, puis est partie travailler à Lisbonne. 
son frère aîné parti dans le cadre d'une coopération à Lisbonne et revenu en France au bout de deux ans.

Si s'écarter du projet migratoire parental peut dans certains cas engendrer une très forte culpabilité, dans l'ensemble, les jeunes d'origine portugaise semblent réussir à se dégager des contraintes du projet migratoire familial : ils partent au Portugal, laissant leurs parents derrière eux... Leurs parents sont dans certains cas tiraillés entre leur profond désir de retour au pays au moment de la retraite et le fait de repartir sans leurs enfants : "Tous les ans c'est pas beaucoup, surtout que mes parents sont vieux $[\ldots] »$, confie une femme portugaise dont le mari «s'est résigné à rester en France puisque c'est le désir des enfants » (ouvrière, Brétigny-sur-Orge). Une autre femme raconte : « Lui [son mari], il voudrait partir tout de suite, mais je ne peux pas laisser mes enfants, en tout cas pas pour le moment...» (employée de maison, Paris) ${ }^{79}$. Pour certains des migrants rencontrés, c'est le projet de vie de leurs enfants qui guidera le choix d'un retour définitif au Portugal au moment de la retraite - les enfants ayant choisi de s'y installer -, ou le choix d'un va-et-vient entre la France et le Portugal, si tel n'est pas le cas. Les notions de « migrations pendulaires » ou « migrations circulatoires » apparaissent plus pertinentes pour définir les pratiques de mobilité spatiale observées chez les migrants portugais. Elles prennent la forme d'un va-et-vient entre le lieu de résidence et le lieu d'origine et non la forme d'une installation définitive ${ }^{80}$. Ces circulations permettent de créer un équilibre entre le retour chez soi et la présence auprès des enfants. Qui plus est, cette solution permet de garder une adresse en France, et le maintien de l'accès à ses infrastructures. Le système social et sanitaire portugais est fréquemment critiqué et constitue une des raisons évoquées pour le choix de la double résidence ${ }^{81}$.

La question des émigrés reste un sujet sensible dans la société portugaise et met en évidence un écart certain entre le rôle symbolique de l'émigré « ambassadeur de la culture portugaise » dans le monde, que lui attribuent certains responsables institutionnels de l'État portugais ${ }^{82}$, et sa culture effective, celle d'un monde rural fermé sur lui-même au temps de l'émigration et perpétuée au sein de l'immigration. Les préjugés à l'égard de l'« emigrante » sont omniprésents

79. Selon LeITE, 1999, p. 306, le retour définitif au Portugal est très rarement le fait d'une décision féminine : «[...] mais c'est surtout au nom de la permanence de l'unité familiale qu'elles revendiquent un projet de sédentarisation au pays d'accueil. »

80. MOROKVASIC et RUDOLPH, dir., 1996.

81. Le sociologue portugais SANTOS, 1997, définit le Portugal comme pays « semipériphérique » dans lequel les insuffisances de l'État en matière de politique sociale sont partiellement comblées par une « société providence » forte.

82. Selon PoInARD, 1988, la « fonction miroir » est en effet un attribut essentiel dans l'immigration. 
dans la société portugaise, se rapportant aux pratiques ostentatoires adoptées lors des retours annuels, mais également à leur manque de capital culturel ${ }^{83}$. « Si un groupe suffisamment large de lusodescendants réussit à maîtriser, dans les deux espaces, les divers segments du capital culturel, les "impressions" sur leurs parents, y compris sur leurs maisons, peuvent quelque peu se modifier», espère le sociologue portugais Sergio Lopes ${ }^{84}$.

Depuis 1999, le secrétariat aux Communautés portugaises ainsi que le secrétariat d'État à la Jeunesse organisent annuellement une « Rencontre mondiale des jeunes portugais et lusodescendants », dont l'objectif est de

« faciliter la rencontre de jeunes portugais et lusodescendants de divers points du monde avec des jeunes et des associations juvéniles résidant au Portugal, autour de sessions de réflexion [...] d'actions de formation, permettant également la rencontre avec des entités officielles et un contact avec la réalité locale ${ }^{85}$ ».

Lors de la rencontre de mai 2001, environ quatre-vingts « lusodescendants » en provenance du Canada, des États-Unis, du Brésil, du Venezuela, de l'Argentine, d'Afrique du Sud, du Mozambique, de Macao, d'Australie, de France, d'Allemagne et de Suisse, tous étudiants, avocats, chercheurs, journalistes, sportifs, se sont rencontrés durant une semaine aux portes de Lisbonne, dans une auberge de jeunesse surplombant le Tage et dont l'imposant pont suspendu dénommé « pont du 25 avril » symbolise aux yeux des institutionnels présents ces «lusodescendants » : ils sont le pont entre le Portugal et leurs pays de résidence respectifs.

Le contact proposé avec « la réalité locale portugaise » se situe à l'opposé de la réalité des villages d'origine, dans lesquels, avant ou après la rencontre, ces jeunes sont allés rendre visite à leur famille. Des conférences thématiques sur la situation socio-économique du pays y étaient proposées : la bonne santé économique du Portugal (taux de chômage inférieur à $5 \%$ ), les performances technologiques et la créativité industrielle (présentation de nouvelles technologies de l'information servant à divulguer les produits adressés « aux Communautés »). L'évolution du Portugal et son « déficit en main-d'œuvre qualifiée ${ }^{86}$ » sont des sujets récurrents. Ces manifestations sont ponctuées par des instants improvisés par les « lusodescendants »: danses et chants populaires portugais, héritage

83. GonÇalves, 1996.

84. LOPES, 1998, p. 87.

85. Fiche d'inscription pour la rencontre, ministère des Affaires étrangères : « [...] uma iniciative que pretende proporcionar o encontro de jovens portugueses e lusodescendentes de vários pontos do mundo com jovens e associações juvenis residentes em Portugal, em redor de sessões de reflexão e trabalho subordinadas a diversos temas, acções de formação, assim como proporcionar o encontro com entidades oficiais e contacto com a realidade local. »

86. Propos d'un conseiller du Premier ministre portugais, Almada (Portugal), mai 2001. 
commun, permettent de rompre avec la formalité des visites très médiatisées dans les palais du Premier ministre et du président de la République.

Un programme intitulé «Estágios Profissionais para Jovens Portugueses e Luso-Descendantes Residentes no Estrangeiro ${ }^{87}$ » a par ailleurs été lancé au cours de l'année 2001 par le ministère des Affaires étrangères portugais (dont dépend le secrétariat d'État aux Communautés portugaises), ainsi que par le ministère du Travail et de la Solidaritée ${ }^{88}$. Dans un contexte où le « rôle des lusodescendants dans l'affirmation et la visibilité du Portugal à l'étranger [est] accru », et où « la valorisation culturelle, académique et professionnelle de ces jeunes » devient nécessaire ${ }^{89}$, ce programme propose aux enfants d'émigrés portugais un stage de neuf mois dans une entreprise portugaise. Les premiers candidats sont originaires du Brésil, du Venezuela et de la France, âgés entre 18 et 30 ans, et sans emploi. L'objectif du programme étant de « préparer à l'insertion professionnelle au Portugal ou au pays d'origine ${ }^{90} »$ des jeunes avec une qualification supérieure, technique ou professionnelle. Ces candidats ont comme motivation première - comme rêve - celle de s'installer au Portugal. Un « retour » aux racines, qui permettent aussi de contourner les difficultés du marché de l'emploi dans le pays de résidence. Cependant, ces descendants de migrants portugais semblent partager entre la réalisation du rêve de « retour »celui de leurs parents avant tout -, le désir de jouer un rôle dans le développement de « leur » pays - ils parlent d'une « mission »- et le sentiment que l'État portugais «achète [leur] double identité ». En dehors de ces programmes très spécifiques adressés à une population susceptible de répondre aux besoins du marché du travail portugais, il semble cependant exister un « décalage important entre l'incitation des politiques à encourager la mobilité et les carences administratives et informatives auxquelles les jeunes se heurtent ${ }^{91} »$. Selon Maria Beatriz Rocha-Trindade, le concept imaginé de retour, comme la fin des difficultés et la fin idyllique du cycle transitoire de déracinement, s'il est un mythe des migrants, il est aussi un prétexte commode des États qui visent de manière simpliste la résolution de problèmes ${ }^{92}$.

Entre une «double culture » qui représente parfois un réel dilemme identitaire, un patriotisme ou encore un pluralisme culturel assumé, les fonctions

87. Stages professionnels pour jeunes portugais et lusodescendants résidant à l'étranger, Diário de Coimbra, 3 mai 2001. L'article annonce l'arrivée de 159 jeunes en provenance de divers pays, et un objectif annuel de 1000 candidats à atteindre.

88. Programme promu par l'institut de l'Emploi et de la Formation professionnelle et la direction générale des Affaires consulaires et des Communautés portugaises.

89. Site Internet du secrétariat d'État aux Communautés portugaises.

90. Site Internet du secrétariat d'État aux Communautés portugaises.

91. Villanova, 2000, p. 143.

92. Rocha-TRINDADE, 1983, p. 95. 
économiques mais surtout identitaires des pratiques de circulation observées laissent entrevoir la possibilité d'une confrontation entre des représentations héritées d'une mémoire collective et la réalité - une confrontation cependant enchevêtrée dans des intérêts nationaux et familiaux.

Des jeunes d'ascendance portugaise et des jeunes portugais de France circulent en puisant dans des ressources valorisantes de l'histoire nationale portugaise, dans des valeurs héritées de parcours migratoires familiaux, ainsi que dans les différentes réalités économiques, culturelles et politiques de l'Union européenne. Leurs pratiques de circulation entre la France et le Portugal se fait en mobilisant des ressources culturelles, identitaires et symboliques plurielles : le bilinguisme, la double résidence (la maison construite « au pays » et les liens avec la famille résidante, mais aussi la maison familiale en France vers laquelle un autre retour est toujours possible), la binationalité (parfois utilisée dans la construction de projets professionnels). Ils utilisent de nouvelles ressources, dont l'émergence relève des politiques étatiques : la liberté de circuler et de s'installer pour tout citoyen européen sur le territoire de l'Union européenne, l'équivalence des diplômes, les programmes d'insertion professionnelle de l'État portugais...

Si ces « lusodescendants » symbolisent d'une certaine manière la figure du citoyen européen caractérisée par le fait de travailler, de s'installer et d'étudier dans n'importe lequel des quinze États membres ${ }^{93}$, ils ne circulent cependant pas entre n'importe quel pays et pour de simples raisons économiques. Le sentiment « d'être européen » émerge chez certains d'entre eux, défini comme un syncrétisme valorisant leur spécificité culturelle ${ }^{94}$. L'idée selon laquelle, parce qu'il n'existe pas de dévalorisation de l'origine portugaise, comme c'est le cas pour les maghrébins dans la société française, il n'existerait pas d'effort d' « autovalorisation collective » chez les jeunes d'origine portugaise ${ }^{95}$, est à nuancer, dans la mesure où le contexte économique et culturel européen semble constituer un cadre favorable à la valorisation de leur identité culturelle. Les discours et pratiques identitaires observés illustrent une volonté de s'inscrire, avec plus ou moins d'autonomie, dans une mémoire collective portugaise quand ils s'affirment «citoyens du monde », ils investissent la dimension cosmopolite de la culture portugaise - et familiale, dont certains cherchent à travers le « retour » à lever les silences.

Irène Dos SANTOS

(janvier 2002).

93. Eurobaromètre, 1997, p. 111.

94. En réponse à la question : « Vous sentez-vous portugais ; de la nationalité du pays où vous résidez; des deux en même temps ; européen au-delà des nationalités ; autres », 1/10 des jeunes enquêtés se sentent européens au-delà de la nationalité. Voir LA BARRE, 1997.

95. Cordeiro, 1989, p. 31. 


\section{LISTE DES RÉFÉRENCES}

Abou-SADA (Georges) et Milet (Hélène), 1986, Générations issues de l'immigration. Mémoires et devenirs, Paris, Arcantères.

ANDERSON (Benedict), 1996, L'Imaginaire national. Réflexions sur l'origine et l'essor du nationalisme, Paris, La Découverte.

Bonnin (Philippe) et Villanova (Roselyne de), dir., 1999, D’une maison à l'autre. Parcours et mobilités résidentielles, Grâne (Drôme), Créaphis.

Bonvalet (Catherine) et Villanova (Roselyne de), 1999, « Immigrés propriétaires ici et là-bas, un système résidentiel ? », in Bonnin et VILlanova, dir., 1999, p. 213-246.

CAllier-Boisvert (Colette), 1999, Soajo entre migrations et mémoire. Études sur une société agro-pastorale à l'identité rénovée, Paris, Centre culturel Calouste Gulbenkian.

Charbit (Yves), Hily (Marie-Antoinette) et Poinard (Michel), 1997, Le Va-et-vient identitaire. Migrants portugais et village d'origine, Paris, Presses universitaires de France-Institut national d'études démographiques.

CordeIro (Albano), 1989, «Le paradoxe de l'immigration portugaise », Hommes et migrations, 1123, juin-juil., p. 25-32.

Cunha (Luís), 2001, A Nação nas malhas da sua identidade. O Estado Novo e a construção da identidade nacional, Porto, Edições Afrontamento.

Eurobaromètre, 1997, Les Jeunes Européens, rapport du 29 juillet, 47.2, pour la Direction générale XXII, «Éducation, formation, jeunesse », INRA.

FELdMAN-BiAnCo (Bela), 1995, « A crianção de uma nação (portuguesa) desterritorializada e a transnacionalização de famílias », Cadernos CERU, sér. 2, 6, p. 89-104.

GALLissot (René), 1993, « Pluralisme culturel en Europe. Identités nationales et identité européenne. De l'intellectuel métis au métissage culturel de masses », in ID., éd., Pluralisme culturel en Europe. Cultures européennes et cultures des diasporas, Paris, L'Harmattan, p. 12-27.

GodinHo (Vitorino Magalhães), 1978, «L'émigration portugaise ( $\mathrm{XV}^{\mathrm{e}}-\mathrm{XX}$ siècles). Une constante structurale et les réponses aux changements du monde », Revista de História Economica e Social, janv.-juin, 1, p. 5-32.

GONÇALVES (Albertino), 1996, Imagens e clivagens. Os residentes face aos emigrantes, Porto, Edições Afrontamento.

LA BARRE (Jorge de), 1997, Jeunes d'origine portugaise en association. On est européen sans le savoir, Paris-Montréal, L'Harmattan.

LEAL (João), 1999, «Saudade, la construction d'un symbole "Caractère national" et identité nationale », Ethnologie française, 2, p. 177-189.

LEAL (J.), 2000, Etnografias portuguesas (1870-1970). Cultura popular e identidade nacional, Lisbonne, Publicações Dom Quixote. 
Leandro (Maria Engrácia), 1995, Au-delà des apparences. Les Portugais face à l'insertion sociale, Paris, L'Harmattan.

LEANDRo (M. E.), 2000, «A construção social da diferença através da acção denominativa. O caso dos jovens portugueses perante as migrações internacionais », Cadernos do Noroeste, série Sociologia, vol. XIII, 1, p. 5-30.

LeITE (Carolina), 1999, «Femmes et enjeux familiaux de la double résidence », in Bonnin et Villanova, dir., 1999, p. 295-312.

LOPES (Sergio), 1998, « Le Portugal et ses émigrés », in Rocha-Trindade et RAVEAU, org., 1998, p. 53-89.

LourenÇO (Eduardo), 1994, «L'Europe dans l'imaginaire portugais », Peuples méditerranéens, 66, p. 211-218.

Lourenço (E.), 1997, Mythologie de la saudade, Paris, Chandeigne.

Marques (A. H. de Oliveira), 1998, Histoire du Portugal et de son empire colonial, Paris, Karthala.

Mattoso (José), 1998, A Identidade nacional, Lisbonne, Gradiva (Fundação Mário Soares).

Melo (Daniel), 2001, Salazarismo e cultura popular (1933-1958), Lisbonne, Universidade de Lisboa.

MonteIro (Paulo Filipe), 1994, Emigração o eterno mito do retorno, Oeiras, Celta Editora.

Morokvasic (Mijana) et RudOlPH (Hedwig), dir., 1996, Migrants. Les nouvelles mobilités en Europe, Paris, L'Harmattan.

MuÑoz (Marie-Claude), 1998, «Les fêtes au Portugal », Informations sociales, 70, p. 42-51.

MuÑOz (M.-C.), 1999, « Des “Tos" aux "Luso-descendants”. L'identité des enfants d'immigrés portugais revisitée », Latitudes. Cahiers lusophones, 5, p. 7-8.

NoIRIEL (Gérard), 2001, État, nation et immigration. Vers une histoire du pouvoir, Paris, Belin.

OrIOL (Michel), 1985, « Du navigateur au prolétaire. L'histoire comme ressource identitaire dans la diaspora portugaise », Migrations et Méditerranée. Peuples méditerranéens, 31-32, avr.-sept., p. 203-215.

PoInARD (Michel), 1988, «La politique d'un pays d'origine : le Portugal », Revue européenne des migrations internationales, vol. IV, 1-2, p. 187-202.

Portugal BRANCO (Jorge de), 1996, « Breve caracterização demográfica dos portugueses radicados em França », Cadernos do Noroeste, vol. IX, 1, p. 77-92.

Portugal Branco (J. de), 1999, «L'émigration et son contexte. Quelques éléments de réflexion sur le Portugal », Latitudes. Cahiers lusophones, 5, p. 29-33.

Portugal Branco (J. de), 2001, «Comunidade portuguesa radicada em França em 1999 », Recensement INSEE 1999 acquis par l'ambassade du Portugal de France (polycopié).

Portugal Branco (Jorge de), Ribeiro (Isabel Amorim) et Villanova (Roselyne de), 1997, « Jeunes issus de l'immigration en Région parisienne », Hommes et migrations, 1210, nov.-déc., p. 73-77.

Rato (Helena), 2001, «O retorno dos emigrantes », Janus. Anuário de Relações Exteriores, Lisbonne, Universidade Autónoma, p. 164-165.

Rocha-Trindade (Maria-Beatriz), 1983, «O regresso imaginado », Nação e Defesa, separata out-dez., 28, Instituto da Defesa Nacional (Lisbonne), p. 87-97. 
Rocha-TRINDADE (M.-B.), 1986, « Longitudinalmente diferente ou o discurso polémico de luso-descendentes », Análise Social, vol. XXII, 92-93, p. 609-618.

RochA-Trindade (M.-B.), 1987, « As micropátrias do interior português », Análise Social, vol. XXIII, 98, p. 721-732.

Rocha-Trindade (Maria-Beatriz) et Raveau (François H. M.), org., 1998, Présence portugaise en France, Lisbonne, Universidade Aberta, CEMRI.

Santos (Bonaventura de Sousa), 1997, Pela Mão de Alice o social e o político na pósmodernidade, $1^{\text {re }}$ éd. Porto, 1994, ici Porto, Edições Afrontamento.

SERRÃo (Joel), 1982, A Emigração portuguesa, $1^{\text {re }}$ éd. Lisbonne, 1972, ici Lisbonne, Livros Horizonte.

VAlensi (Lucette), 1992, Fables de la mémoire. La glorieuse bataille des trois rois, Paris, Seuil.

ViLlanova (Roselyne de), 1986, « La langue du "retour" ou le retour de la langue. Les pratiques d'alternance linguistique dans la famille portugaise émigrée en France », in AbOU-SADA et Milet, 1986, p. 169-179.

Villanova (R. de), 1999, «Digressions sur l'indicible, le secret et la séparation dans l'émigration », Sigila, printemps-été, p. 21-31.

Villanova (R. de), 2000, « Mobilité géographique, intégration et double résidence des jeunes issus de l'immigration portugaise », in VARINE (Béatrice de), dir., Lieux de vie et circulations des Portugais de France, Paris, Interaction France-Portugal, p. 133-144.

Villanova (Roselyne de), LeIte (Caroline) et Raposo (Isabel), 1994, Maisons de rêve au Portugal, Grâne (Drôme), Créaphis.

Zonabend (Françoise), 1980, «Prénom et identité », in DupÂQuiER (Jacques), BIDEAU (Alain) et Ducreux (Marie-Élisabeth), dir., Le Prénom, mode et histoire. Entretiens de Malher, Paris, Éditions de l'École des hautes études en sciences sociales (Recherches d'histoire et de sciences sociales), p. 23-27. 